\title{
EFEITO DA PRIMAVERA E VERÃO NO QUADRO ESPERMÁTICO DE TOUROS NELORE
}

Willian Mituzi Tateisi, Camila Dutra de Souza, Gabriela Figueredo Cornacini, Talita Cavichioli Sebastião, Fernanda Luisa Guinossi Barbosa Deak, Marcelo George Mungai Chacur

Universidade do Oeste Paulista, curso de Medicina Veterinária, Presidente Prudente, SP. Agência de fomento: CNPq

E-mail: willian tateisi@hotmail.com

\section{RESUMO}

O objetivo dessa pesquisa foi analisar o perfil seminal, através do exame andrológico, de touros da raça Nelore nas estações da primavera e verão, criados extensivamente, para fins de construir critérios de seleção reprodutiva. Foram utilizados 20 touros da raça Nelore durante 6 meses, acompanhando as estações primavera e verão, sendo 2 coletas por estação. Mensurados os fatores climáticos e colhido sêmen para análise microscópica do ejaculado, determinando motilidade progressiva (0 a 100\%), vigor ( 1 a 5) e turbilhão (0 a 5 ) e morfologia espermática Os dados foram submetidos à análise de variância com posterior Teste de Tukey a 5\%. Conclui-se que o touro nelore no verão ejacula maior volume $(5.91 \pm 2.98 \mathrm{~mL})$ e tem maior motilidade espermática (70.75 $\pm 17.64 \%)$, porem tem mais defeitos espermáticos maiores (18.32 $\pm 9.07 \%)$ e totais $(23.80 \pm 10.89 \%)$ que a estação da primavera. Mostrando que os fatores climáticos estão diretamente ligados à qualidade do sêmen.

Palavras-chave: Fatores climáticos; Sêmen; Zebu; estacionalidade

\section{EFFECT OF SPRING AND SUMMER ON THE SPERMATIC PROFILE OF NELORE BULLS}

\begin{abstract}
The objective of the research was to analyze the seminal profile, through the andrological examination, of Nelore bulls in the spring and summer seasons, created extensively, for the purpose of constructing reproductive selection criteria. Twenty four Nelore bulls aged 24 months, were used for 6 months, following the spring and summer seasons, two in spring and two in summer. Measured the climatic factors and the semen samples was to analyze, determining sperm motility progressive ( 0 to $100 \%$ ), vigor ( 1 to 5 ) and mass motion ( 0 to 5 ) and sperm morphology. The data were submitted to analysis of variance with a Tukey test at 5 . It is concluded that the nelore bull in summer ejaculates larger volume $(5.91 \pm 2.98 \mathrm{~mL})$ and has greater sperm motility $(70.75 \pm 17.64 \%)$, but has larger sperm defects $(18.32 \pm 9.07 \%)$ and total $(23.80 \pm 10.89 \%)$ sperm defects than the spring. Showing that the climatic factors are directly linked to the quality of the semen.
\end{abstract}

Keywords: Climatic factors; Semen; Zebu bull; Seasonality

\section{INTRODUÇÃO}

Levando em conta a predominância da criação extensiva e a monta natural, torna-se necessária a investigação dos fatores climáticos e dos efeitos das estações do ano sobre as características seminais de touros mantidos no campo (OLIVEIRA et al., 2006). A baixa eficiência reprodutiva em touros pode ser causada por mudanças climáticas que afetam a gametogênese (VALE FILHO, 2001). É necessário haver uma melhoria genética dos rebanhos para identificar reprodutores superiores, aumentando a oferta e o lucro com touros (DIAS et al., 2007). 
Galina e Arthur (1991) relataram que a baixa qualidade do sêmen em alguns animais pode ocorrer por causa do desconforto destes perante temperaturas elevadas. Em outra pesquisa, a temperatura testicular em touros deve ser 4 a $5^{\circ} \mathrm{C}$ abaixo da temperatura corporal para que ocorra espermatogênese normal (COULTER, 1988).

Segundo Kastelic et al. (2001), uma moderada elevação na temperatura testicular em touros reduz drasticamente a produção espermática, a motilidade progressiva, a quantidade de espermatozoides vivos por ejaculado e aumenta a porcentagem de espermatozoides morfologicamente anormais.

A alta temperatura da maioria dos ambientes tropicais afeta os processos reprodutivos diretamente e indiretamente através do estresse térmico ou calórico. Nos machos bovinos, altas temperaturas podem provocar esterilidade estival, degeneração do epitélio germinativo, abaixamento da produção de sêmen e queda da fertilidade (MEDEIROS; VIEIRA, 1997).

Climas quentes afetam a produção espermática em ruminantes devido à redução na taxa de crescimento corporal e testicular correlacionada com a disponibilidade nutricional. Esta reduzida taxa de crescimento do testículo é acompanhada da diminuição de espermatócitos e maturação de espermátides principalmente devido à redução da capacidade esteroidogênica das células de Leydig, levando a uma significativa redução da produção espermática (COSTA E SILVA, 2004).

O presente trabalho justifica-se para colaborar com dados inerentes ao sêmen de Nelores devido a influência dos fatores climáticos na reprodução. Desta forma, o objetivo da presente pesquisa foi analisar o perfil seminal, através do exame andrológico, de touros da raça Nelore nas estações primavera e verão, criados extensivamente, para fins de monta natural.

\section{MATERIAL E MÉTODOS}

Presente pesquisa foi aprovada pelo Comitê de Ética de Uso de Animais em Experimentação/Unoeste pelo protocolo no 3479.

Foram utilizados 20 touros da raça Nelore com idades de 24 meses, de uma propriedade do município de Anaurilândia - MS, durante 6 meses, acompanhando as estações da primavera e verão do ano de $2015 / 2016$. Os animais ficaram mantidos durante o projeto em pasto de Urochloa decumbens com acesso à água e mistura mineral à vontade, em sistema extensivo de produção.

Foram mensurados os fatores climáticos: temperatura ambiente, umidade relativa do ar, temperatura de bulbo negro seco e temperatura do globo, nos dias de colheita a cada hora, entre 8:00h e 12:00h, com o termômetro de globo portátil digital (modelo HT-30, InstruTemp). Os dados do índice pluviométrico foram verificados nas estações primavera e verão através do Instituto Nacional de Meteorologia (INMET).

Foram realizadas quatro colheitas de sêmen, sendo duas na primavera (colheitas 1 e 2) e duas no verão (colheitas 3 e 4), com método de eletroejaculação na modalidade automática (Modelo Eletroejac ${ }^{\circledR}$, Neovet, Brasil), conforme normas do Colégio Brasileiro de Reprodução Animal (CBRA, 2013).

Os ejaculados foram mantidos em banho-maria entre 32 e 37으, para a análise ao microscópio de motilidade espermática progressiva (0 a 100\%), vigor espermático (1 a 5 ) e turbilhão (0 a 5). Ainda foram considerados, o volume do ejaculado ( $\mathrm{mL}$ ), a cor e o aspecto. Em uma solução de formol salino tamponado, o sêmen foi diluído em uma proporção de 1:100, para posterior contagem em câmara de Neubauer. A morfologia espermática foi analisada em microscopia de contraste de fase, levando-se em consideração 200 espermatozoides. As análises do sêmen foram realizadas conforme normas do Colégio Brasileiro de Reprodução Animal (CBRA, 2013). 
Os dados foram submetidos à análise de variância com posterior Teste de Tukey a 5\%, com comparação dos dados entre as colheitas e as estações do ano primavera e verão, com o programa estatístico BioEstat ${ }^{\circledR}$ 5.3.

\section{RESULTADOS E DISCUSSÃO}

O parâmetro volume do ejaculado apresentou diferença $(P<0,05)$ entre as colheitas 1 e 4; e entre as estações primavera e verão, aonde se obteve maior volume no verão e não apresentou diferença $(P>0,05)$ entre as colheitas 1 e $2 ; 1$ e $3 ; 2$ e 3 ; e 3 e 4.

O parâmetro motilidade apresentou diferença $(P<0,05)$ entre as colheitas 1 e 3 , houve motilidade maior na colheita 3 , mas não apresentou diferença $(P>0,05)$ entre as colheitas (1 e 2; 2 e 3; 3 e 4; e entre as estações da primavera e verão). Os parâmetros vigor e turbilhão não apresentaram diferenças $(P>0,05)$ entre colheitas.

$O$ parâmetro defeitos espermáticos menores não apresentou diferença $(P>0,05)$ entre as colheitas 1 e 2; 1 e 4; e 3 e 4, mas apresentou diferença $(P<0,05)$ entre as colheitas 2 e 3; e entre as estações da primavera e verão, com maior porcentagem de defeitos na primavera.

$O$ parâmetro defeitos espermáticos maiores não apresentou diferença $(P>0,05)$ entre as colheitas 1 e 2; e 2 e 3, mas apresentou diferença $(P<0,05)$ entre as colheitas 1 e 3 ; 1 e 4; e entre as estações houve maior porcentagem no verão. $O$ parâmetro defeitos espermáticos totais não apresentou diferença $(P>0,05)$ entre as colheitas 1,2 e 3 , mas apresentou diferença $(P<0,05)$ para as colheitas 1, 2 e 3, em relação a colheita 4; e entre as estações da primavera e verão, com maior porcentagem de defeitos totais no verão.

Tabela 1. Parâmetros quantitativos e qualitativos (Média \pm desvio padrão) do sêmen entre as coletas 1, 2, 3 e 4 e entre as estações primavera e verão.

\begin{tabular}{ccccc|cc} 
& Coleta 1 & Coleta 2 & Coleta 3 & Coleta 4 & Primavera & Verão \\
\hline Volume & $3.65 \pm 2.40 \mathrm{~B}$ & $4.80 \pm 2.33 \mathrm{AB}$ & $5.85 \pm 3.18 \mathrm{AB}$ & $5.97 \pm 2.14 \mathrm{~A}$ & $4.22 \pm 2.41 \mathrm{~B}$ & $5.91 \pm 2.68 \mathrm{~A}$ \\
Motilidade & $54.50 \pm 14.68 \mathrm{~B}$ & $69.44 \pm 14.02$ & $70.75 \pm 17.64 \mathrm{~A}$ & $68.94 \pm 17.84 \mathrm{AB}$ & $61.57 \pm 16.07 \mathrm{~A}$ & $69.87 \pm 17.52 \mathrm{~A}$ \\
$\begin{array}{c}\text { Vigor } \\
\text { Turbilhão }\end{array}$ & $2.50 \pm 0.88 \mathrm{~A}$ & $3.05 \pm 0.72 \mathrm{~A}$ & $3.05 \pm 0.88 \mathrm{~A}$ & $2.89 \pm 0.80 \mathrm{~A}$ & $2.76 \pm 0.85 \mathrm{~A}$ & $2.97 \pm 0.84 \mathrm{~A}$ \\
$\begin{array}{c}\text { Defeitos } \\
\text { menores }\end{array}$ & $9.53 \pm 4.12 \mathrm{~A}$ & $\mathbf{8 . 0 4 5 \pm 4 . 8 9 \mathrm { A }}$ & $3.74 \pm 2.05 \mathrm{~B}$ & $7.32 \pm 4.62 \mathrm{AB}$ & $8.83 \pm 4.51 \mathrm{~A}$ & $5.48 \pm 3.94 \mathrm{~B}$ \\
$\begin{array}{c}\text { Defeitos } \\
\text { maiores }\end{array}$ & $6.65 \pm 4.81 \mathrm{C}$ & $7.09 \pm 5.27 \mathrm{BC}$ & $13.77 \pm 6.81 \mathrm{~B}$ & $23.10 \pm 8.82 \mathrm{~A}$ & $6.86 \pm 4.97 \mathrm{~B}$ & $18.32 \pm 9.07 \mathrm{~A}$ \\
$\begin{array}{c}\text { Defeitos } \\
\text { totais }\end{array}$ & $16.18 \pm 6.85 \mathrm{~B}$ & $15.14 \pm 8.63 \mathrm{~B}$ & $17.51 \pm 7.10 \mathrm{~B}$ & $30.42 \pm 10.34 \mathrm{~A}$ & $15.69 \pm 7.65 \mathrm{~B}$ & $23.80 \pm 10.89 \mathrm{~A}$ \\
\hline
\end{tabular}

Letras diferentes na linha diferem entre si pelo teste de Tukey $(\mathrm{P}<0.05)$

$O$ defeito espermático maior de patologia de cabeça não apresentou diferença $(P>0,05)$ entre as coletas $1,2,3$ e 4 , mas apresentou diferença $(P<0.05)$ entre as estações primavera e verão, com o valor da estação do verão mais alta.

$O$ defeito espermático maior de acrossomo não apresentou diferença ( $P>0.05)$ entre as coletas 1 , 2 e 3 , mas apresentou diferença $(P<0.05)$ das coletas 1 , 2 e 3 em relação a coleta 4 e entre as estações primavera e verão, com os valores da estação do verão mais elevados.

Os defeitos espermáticos maiores de gota proximal e patologia de cauda não apresentaram diferença $(P>0.05)$ em nenhuma coleta e em nenhuma estação. 
O defeito espermático maior de defeito de peça intermediária não apresentou diferença $(P>0.05)$ entre as coletas 1 e 2 , e 3 e 4 , apresentando diferença $(P<0.05)$ das coletas 1 e 2 em relação à 3 e 4, e nas estações primavera e verão, com os valores da estação do verão mais alta.

$O$ defeito espermático menor de patologia de cabeça não apresentou diferença $(P>0.05)$ entre as coletas 1, 2, 3 e 4, mas apresentou diferença $(P<0.05)$ entre as estações primavera e verão, com a média da estação da primavera mais alto.

$O$ defeito espermático menor de gota distal não apresentou diferença $(P>0.05)$ em nenhuma coleta e em nenhuma estação.

$O$ defeito espermático menor de patologia de cauda não apresentou diferença $(P>0.05)$ entre as coletas 1 e 2, 1 e 4, 2 e 3, 2 e 4, e 3 e 4, mas apresentou diferença $(P<0.05)$ entre as coletas 1 e 3 e entre as estações primavera e verão, com o valor da estação da primavera mais elevado.

Tabela 2. Defeitos espermáticos maiores e menores (Média \pm desvio padrão) entre as coletas 1, 2 , 3 e 4 e entre as estações primavera e verão.

\begin{tabular}{|c|c|c|c|c|c|c|}
\hline & Coleta 1 & Coleta 2 & Coleta 3 & Coleta 4. & Primavera & Verão \\
\hline \multicolumn{7}{|l|}{ Defeitos Maiores } \\
\hline \multicolumn{7}{|l|}{$\begin{array}{c}\text { Patologia de } \\
\text { Cabeca }\end{array}$} \\
\hline \multirow{2}{*}{$\begin{array}{c}\text { Acrossomo } \\
\text { Gota Proximal } \\
\text { Defeito de PI } \\
\text { Patologia de } \\
\text { Cauda } \\
\end{array}$} & $\begin{array}{l}0.46 \pm 1.24 \mathrm{~B} \\
0.23 \pm 0.53 \mathrm{~A} \\
3.33 \pm 3.60 \mathrm{~B}\end{array}$ & $\begin{array}{l}0.67 \pm 1.32 \mathrm{~B} \\
0.09 \pm 0.28 \mathrm{~A} \\
2.79 \pm 2.96 \mathrm{~B}\end{array}$ & $\begin{array}{l}2.42 \pm 3.01 \mathrm{~B} \\
0.69 \pm 1.91 \mathrm{~A} \\
6.78 \pm 3.74 \mathrm{~A}\end{array}$ & $\begin{array}{l}8.05 \pm 6.82 \mathrm{~A} \\
0.37 \pm 0.70 \mathrm{~A} \\
9.78 \pm 5.33 \mathrm{~A}\end{array}$ & $\begin{array}{l}0.56 \pm 1.27 \mathrm{~B} \\
0.16 \pm 0.42 \mathrm{~A} \\
3.06 \pm 3.27 \mathrm{~B}\end{array}$ & $\begin{array}{l}5.24 \pm 5.93 \mathrm{~A} \\
0.53 \pm 1.43 \mathrm{~A} \\
8.28 \pm 4.79 \mathrm{~A}\end{array}$ \\
\hline & $2.09 \pm 2.12 \mathrm{~A}$ & $2.11 \pm 2.07 \mathrm{~A}$ & $2.47 \pm 2.57 \mathrm{~A}$ & $1.92 \pm 1.47 \mathrm{~A}$ & $2.10 \pm 2.07 \mathrm{~A}$ & $2.19 \pm 2.09 \mathrm{~A}$ \\
\hline \multicolumn{7}{|c|}{ Defeitos Menores } \\
\hline \multirow{3}{*}{$\begin{array}{c}\text { Patologia de } \\
\text { Cabeça } \\
\text { Gota Distal } \\
\text { Patologia de } \\
\text { Cauda } \\
\end{array}$} & $3.10 \pm 3.14 \mathrm{~A}$ & $3.55 \pm 2.80 \mathrm{~A}$ & $1.65 \pm 1.39 \mathrm{~A}$ & $2.30 \pm 2.00 \mathrm{~A}$ & $3.32+2.95 \mathrm{~A}$ & $1.97 \pm 1.73 \mathrm{~B}$ \\
\hline & $0.29 \pm 1.10 \mathrm{~A}$ & $0.00 \pm 0.00 \mathrm{~A}$ & $0.08 \pm 0.26 \mathrm{~A}$ & $0.14 \pm 0.48 \mathrm{~A}$ & $0.15 \pm 0.78 \mathrm{~A}$ & $0.11 \pm 0.38 \mathrm{~A}$ \\
\hline & $6.34 \pm 4.23 \mathrm{~A}$ & 4.01士4.39 AB & $2.25 \pm 2.10 \mathrm{~B}$ & $4.71 \pm 3.31 \mathrm{AB}$ & $5.17 \pm 4.41 \mathrm{~A}$ & $3.48 \pm 3.00 \mathrm{~B}$ \\
\hline
\end{tabular}

Letras diferentes na linha diferem entre si pelo teste de Tukey $(P<0.05)$

O WBGT não apresentou diferença $(P>0.05)$ entre as coletas 1,2 e 3 , e nas estações primavera e verão, mas apresentou diferença $(P<0.05)$ das coletas 1 , 2 e 3 em relação à 4 que foi mais baixa.

A temperatura ambiente e do globo não apresentaram diferença $(P>0.05)$ entre as coletas 1 e 2,1 e 4, 2 e 3, 2 e 4, e as estações primavera e verão, mas apresentaram diferença $(P<0.05)$ entre as coletas 1 e 3 , e 3 e 4 .

A umidade relativa do ar não apresentou diferença $(P>0.05)$ entre as coletas 1 e 2, 1 e 3, 2 e 3,2 e 4,3 e 4 , e as estações primavera e verão, só apresentou diferença $(P<0.05)$ entre as coletas 1 e 4 , como pode ser visto na tabela 3 .

Tabela 3. Fatores climáticos (Média \pm desvio padrão) entre as coletas 1, 2, 3 e 4 e entre as estações primavera e verão. 


\begin{tabular}{|c|c|c|c|c|c|c|}
\hline & Coltata & Colota 2 & Colta 3 . & Coleta 4 & Pimavera & Verâa \\
\hline WGGT & $24.721 .1 \mathrm{AB}$ & $26.50 .4 \mathrm{~B}$ & $27.911 .0 \mathrm{AB}$ & $21.70 .9 \mathrm{C}$ & $25.51 .8 \mathrm{~A}$ & $24.8+3.4 \mathrm{~A}$ \\
\hline Tempo. Ambiente & $27.943 .2 \mathrm{~B}$ & $31.21 .3 \mathrm{AB}$ & $32.71 .6 \mathrm{~A}$ & $27.401 .8 \mathrm{~B}$ & $29.42 .9 \mathrm{~A}$ & $30.043 .0 \mathrm{~A}$ \\
\hline Temp. Globo & $280.0+3.3 \mathrm{~B}$ & 31.6t1.0 AB & $33.8 \pm 2.6 \mathrm{~A}$ & $26.71 .2 \mathrm{~B}$ & $29.853 .0 \mathrm{~A}$ & $30.34+2 \mathrm{~A}$ \\
\hline $\begin{array}{l}\text { Umloade } \\
\text { Relativa do ar }\end{array}$ & $68.389 .7 \mathrm{~A}$ & $53.7 \mathrm{Ag} . \mathrm{AB}$ & 56.7AA. AB & 50.012.78 & $61.7 \pm 11.5 \mathrm{~A}$ & $53.34+8 \mathrm{~A}$ \\
\hline
\end{tabular}

Letras diferentes na linha diferem entre si pelo teste de Tukey $(P<0.05)$.

\section{DISCUSSÃO}

Chacur et al. (2014) concluiu que a morfologia espermática foi influenciada pela época seca com redução dos defeitos maiores e totais nas raças Nelore. Assim como mostra a tabela 1 que tem menor porcentagem de defeitos maiores e totais na primavera.

Souza et al. (2015) encontrou que o aumento da temperatura ambiente influencia na elevação dos defeitos espermáticos menores e maiores, mas conforme os resultados da tabela 2 observa-se que em temperatura ambiente alta (verão) tem aumento dos defeitos espermáticos maiores apenas.

Adicionalmente, a média do índice pluviométrico na primavera foi $128 \mathrm{~mm}$ e no verão foi $173 \mathrm{~mm}$, o que está intimamente relacionado à qualidade da pastagem e ao conforto térmico.

\section{CONCLUSÃO}

Nas condições experimentais, conclui-se que as estações do ano influenciam na qualidade do sêmen em touros Nelore, criados extensivamente. No verão, o volume e a motilidade do sêmen foram superiores, em relação a primavera. No verão, houve maior presença de defeitos espermáticos maiores e totais. Os touros foram considerados aptos no exame andrológico para fins de monta natural nas duas estações do ano estudadas.

Agradecimentos: CNPq, UNOESTE e Prof. Dr. Carlos Vianna.

\section{REFERÊNCIAS}

CHACUR, M.G.M; MIZUSAKI, K.T; SANTOS, F.H.; CESARE, A.G.; GABRIEL FILHO, L.R.A.; OBA, E.; RAMOS, A.A. Influência da estação do ano nas características do sêmen e na concentração de hormônios em touros Nelore e Simental. Arquivo Brasileiro de Medicina Veterinária e Zootecnia, v. 64, n. 3, p. 540-546, 2012. https://doi.org/10.1590/S0102-09352012000300003

CHACUR, M.G.M.; DOS REIS, J.D.A.; TAVARES, L.S.; SANCHES, K.; GUABERTO, L.; ALVES, V.C.; OBA, E.; RAMOS. A.A. Influência das épocas do ano na morfometria testicular e epididimária, características do sêmen e proteínas do sêmen em SDS-PAGE em zebus e taurinos. Acta Scientiae Veterinariae, v.42, p.1174, 2014.

Colégio Brasileiro de Reprodução Animal (CBRA). Procedimentos para exame andrológico e avaliação de sêmen animal. Belo Horizonte, Brasil. 2013. 
COSTA E SILVA, E. V. Estresse e Manejo reprodutivo de bovinos de corte: problemas e soluções. IV Simpósio de produção de gado de corte, 2004, Viçosa. IV Simcorte. Viçosa: Universidade Federal de Viçosa, 2004. p. 459-484.

COULTER, G. H. Thermography end bull testes. In: PROCEEDINGS OF DE 12Th TECHNICAL CONFERENCE ON ARTIFICIAL INSEMINATION. National Association Animal Breeding, p.58-63. 1988.

DIAS, J.C.; ANDRADE, V.J.; VALE FILHO, V.R; PEREIRA, J. C.C. Caracterização andrológica de touros Nelore criados extensivamente em Mato Grosso do Sul, Brasil. Veterinária Notícias, v.13, p. 39-46, 2007.

GALINA, C. S.; ARTHUR, G. H. Review of cattle reproduction in tropics. Part 6. The Male. Animal Breeding Abstracts, v.59, p.403-412, 1991.

HAFEZ, E.S.E.; HAFEZ, B. Reprodução animal. 7.ed. São Paulo: Manole. 2004. 513p.

KASTELIC, J. P.; COOK, R. B.; COULTER, G. H. Contribution of the scrotum, testes, and testicular artery to scrotal/testicular thermoregulation in bulls at two ambient temperatures. Animal Reproduction Science, v.45, p.255-261, 1997. https://doi.org/10.1016/S0378-4320(96)01587-4

MEDEIROS, L. F. D; VIEIRA, D. H. Apostila de Bioclimatologia Animal, 1997. Disponível em: http://www.iz.ufrrj.br/zootecnia_draa/Biblioteca/Fernando/Apostila\%20de\%20Bioclimatologia\%2 Ol.pdf.

OLIVEIRA, K. M.; DUARTE, A. M.; NASCIMENTO, M. R. B. M. Influência das estações seca e chuvosa sobre as características seminais de touros das raças Nelore, Gir e Holandês criados à pasto. Veterinária Notícias, v. 12, n. 2, p. 145-151, 2006.

SOUZA, C. D., CHACUR, M. G. M., RUEDIGER, F. R., ANDRADE, I. B., SOUZA CARTOCCI, J., BASTOS, G. P., \& OBA, E. Influência dos fatores climáticos no sêmen de touros Nelore (Bos taurus indicus). Colloquium Agrariae, v. 11, n. Especial, p. 2015. https://doi.org/10.5747/ca.2015.v11.nesp.000158

VALE FILHO, V.R. Subfertilidade em touros: parâmetros para avaliação andrológica e conceituação geral. Cad. Tec. Vet. Zootec., n.35, p.81-87, 2001. 